Revista Digital Año 8. No 10 - Año 2017. -pág. 1--151

ISSN 1853-1393

Resistencia. Chaco. Argentina - 2017

\title{
INVESTIGAR NARRATIVAMENTE EN EDUCACIÓN FÍSICA CON RELATOS CORPORALES
}

\section{RESEARCHING NARRATIVELY WITH BODIES STORIES IN PHYSICAL EDUCATION}

\author{
María Esther Prados Megías ${ }^{1}$ - José Ignacio Rivas Flores ${ }^{2}$
}

Fecha de recepción: 19-12-2016

Fecha de aceptación y versión final: 24-04-2017

\begin{abstract}
Resumen:
El cuerpo y todo lo que ello significa en las relaciones humanas está de moda. Sin embargo, la sociedad actual sigue presentándonos lo corporal como una cuestión mecanizada, mercantilizada y cosificada. Los diferentes discursos mediáticos y los modelos corporales hegemónicos, tanto en espacios culturales como educativos, se filtran en nuestra sensorialidad como modelos a reproducir. De ahí que acceder a la experiencia de las personas a través de sus relatos, de sus narraciones y biografías corporales, nos brinden un modo de comprender y entender cómo las personas viven sus cuerpos a través del movimiento. El enfoque y posicionamiento de la investigación narrativa puede darnos claves para incorporar y considerar que investigar, sobre todo en el ámbito de lo corporal en educación, implica un compromiso social, político y ético que no es ajeno a las personas, sobre todo porque atañe a los más íntimo, al cuerpo, ese límite entre lo privado y lo público, entre lo expresado y lo "vivido-sentido". A través de los relatos accedemos al imaginario que los y las estudiantes construyen acerca de la idea de ser educadores o educadoras del movimiento, ya que en sus historias se pone de manifiesto esquemas prácticos para la toma de decisiones, tanto en su formación como en su futura profesión. Los relatos y performances del alumnado dejan entrever elementos que nos permiten hacer del cuerpo un espacio de convergencia discursiva en torno a lo que hemos denominado "cuerpos dietéticos, cuerpos sufridos, cuerpos imaginados y cuerpos lenguajeados".
\end{abstract}

Palabras-clave: educación física, narrativa, relatos corporales, formación inicial

\begin{abstract}
:
The body and everything that it means in human relationships is fashionable. However, today's society continues to present us the corporeal as a mechanized, commodified and reified issue. The different media discourses and the hegemonic corporal models, both in cultural and educational spaces, are filtered in our sensoriality as models to be reproduced. Hence, to access to the experience of people through their accounts, their stories and body biographies, provide us with a way to understand and understand how people live their bodies through movement. The focus and positioning of the narrative inquiry can give us clues to incorporate and consider that researching, especially in the field of the body in education, implies a social, political and ethical commitment that is not alien to people, especially because it concerns the most intimate, the body, that boundary between the private and the public, between the expressed and the "lived-sense". Through stories we access the imaginary that the students build about the idea of being educators of the movement, since in their stories practical schemes for decision making are shown, both in their formation and in their future profession. The stories and performances of the students allow us to
\end{abstract}

\footnotetext{
${ }^{1}$ Profesora Titular Universidad. Área de Didáctica de la Expresión Corporal. Departamento de Educación. Doctora en Antropología Social y Cultural. Profesora del Sistema Consciente para la Técnica del Movimiento. Universidad de Almería (España). C/ Sierra de Gredos, 23, ático B; eprados@ual.es; +34 690729683.

${ }^{2}$ Facultad de Ciencias de la educación. Universidad de Málaga (España). C/ Dante, 44, 29190 Málaga, Catedrático del área de Didáctica y Organización Escolar. Doctor en ciencias de la educación. Coordinador del grupo de Investigación PROCIE (profesorado, comunicación e investigación educativa), del Plan Andaluz de Investigación de la Junta de Andalucía.
} 
glimpse elements that let us to make the body a space of discursive convergence in what we have called "dietary bodies, suffered bodies, imagined bodies and languaged bodies".

Key words: physical education, narrative, bodies stories, initial training

Aunque relativamente recientes, son ya extensos en el tiempo los estudios que diferentes autores y autoras han ido aportando sobre el sentido y necesidad de realizar investigaciones desde el enfoque narrativo al campo de la educación física y las ciencias de deporte (Barbero, 2006; De Mattos, 2013; Devís, 2001, 2004; Eisenberg, 2007; Fernández Balboa, 2014; López Pastor, 2004; Martínez, 2016; Mourao, 2006; Pavía, 2001; Prados, 2004; 2017; Smith, 1999, 2002, 2005, 2006, 2007, 2008a, 2008b, 2009a, 2009b; Sparkes, 1999, 2002, 2003, 2005, 2007, 2008, 2009).

Si bien es cierto que este enfoque ha tenido poco eco en este campo fundamentalmente por la complejidad y cambio de concepción que implica considerar el cuerpo y la motricidad humana desde paradigmas cualitativos-, no lo es menos el hecho de que nos permite profundizar y ampliar la mirada sobre los significados que el cuerpo en movimiento adquiere en la sociedad actual desde la experiencia y subjetividad de las personas. En el ámbito de la cotidianeidad de las personas, el cuerpo -lo corporal, lo deportivo, la salud corporal, etc.,- nos adentra en una necesidad de comprender qué hacemos y cómo vivimos todo lo relacionado con él. Aún con la gran cantidad de avances médicos, deportivos, dietéticos... que nos invaden los canales de información, vivimos en una sociedad que enferma de forma significativa (a veces con síndromes desconocidos hasta el momento), que aumenta los síntomas de cronicidad y dolencias, que vive con la lacra de la obesidad, el estrés, enfermedades mentales, desajustes corporales, etc. ligado a una obsesión creciente por la imagen corporal.

En el ámbito de lo deportivo, además de hablar de éxitos, records, resultados, hazañas deportivas, resultados y competiciones, es necesario igualmente conocer e investigar acerca de abandonos, retiradas, frustraciones, lesiones, soledades, olvidos... Hemos desarrollado una imagen sesgada del deporte, que se mira desde la alta competición, las hazañas, y la excelencia. Podemos decir que el deporte desarrolla una perspectiva congruente con la dinámica social neoliberal, que mira solo desde la competitividad, le éxito y la excelencia.

Desde el ámbito educativo esta perspectiva es relevante, ya que de alguna forma reduce la educación física a esta perspectiva sesgada, buscando seleccionar excelentes deportistas y no formar ciudadanos responsables, de sí mismos, de su salud y su cuerpo y su relación con los otros. Es urgente reflexionar acerca de la falta de sensibilidad y preocupación por incorporar en las escuelas, no ya lo deportivo, sino una educación corporal que trascienda y se haga transversal al resto de contenidos y que vinculen el bienestar afectivo, sensorial, perceptivo, mejora de la atención, escucha, postura, respiración, lenguaje corporal y artístico como cuestiones vinculantes al hecho educativo.

El cuerpo y todo lo que ello significa en las relaciones humanas está de moda. Sin embargo, la sociedad actual sigue presentándonos lo corporal como una cuestión mecanizada, mercantilizada y cosificada. Los diferentes discursos mediáticos y los modelos corporales hegemónicos, tanto en espacios culturales como educativos, se filtran en nuestra sensorialidad como modelos a reproducir. De ahí que acceder a la experiencia de las personas a través de sus relatos, de sus narraciones y biografías corporales, nos brinda un modo de comprender y entender cómo las personas viven sus cuerpos a través del movimiento. 


\section{El sentido de la Narrativa en el ámbito corporal}

La investigación narrativa, como campo de estudio y como planteamiento metodológico, se encuentra suficientemente asentado en el panorama científico en Ciencias Sociales, y en Ciencias de la Educación en particular. (Hernández, 2011; Rivas, 2009). Esto no significa que siempre mantenga una misma perspectiva o que se utilice de la misma manera, lo cual hace necesario establecer la posición en la que nos situamos. Si bien en muchos casos es pensada sólo como una herramienta metodológica, desde nuestra perspectiva la investigación narrativa supone una opción epistemológica, ontológica, ética, ideológica y política (Rivas, 2007, 2009, 2012; Clandinin, 2013). Investigar narrativamente no solo es una forma de construir conocimiento, sino que es conocimiento. Por tanto, es a la vez forma y contenido. Lo cual le sitúa en una perspectiva peculiar que incorpora la capacidad de transformación. El relato construye la realidad, y los sujetos nos constituimos en tanto que participamos de relatos. Por tanto, participar en la construcción de un relato es, también, formar parte del proceso de transformación social y educativa.

En el terreno del cuerpo, en relación a los relatos hegemónicos que actúan socialmente, esta posición es relevante. Este es el relato de una sociedad altamente cualificada, competitiva, estandarizada, tecnológica y globalizada, en el que el sentido de lo físico, de lo corporal, se sitúan en parámetros ajenos al propio sujeto. Por tanto, se invisibiliza, e incluso se niega, se cosifica y unifica la experiencia de las personas, así como sus procesos de formación y conocimiento. Se hace necesario y urgente, plantear otro relato del cuerpo que coloque al sujeto en el centro, así como su relación con el mundo, desde una posición crítica y emancipadora. El enfoque y posicionamiento de la investigación narrativa puede darnos claves para incorporar y considerar el compromiso social, político y ético que representa. En este caso es mucho más patente en tanto que atañe a los más íntimo, al cuerpo, ese límite entre lo privado y lo público, entre lo expresado y lo "vivido-sentido".

Para este cambio de mirada hay que destacar dos cuestiones básicas. La primera tiene que ver con lo que Berry (2008) denomina experiencias particulares 0 pequeñas historias que están siempre conectadas a los grandes relatos o narrativas sociales y que representan un desafío a las narrativas hegemónicas. Son las pequeñas historias las que sacan a la luz cuestiones que nos hablan de pluralidad, diversidad y multiplicidad y, por tanto, nos ayuda a ponerlas en relación con las otras más globales. De este modo es posible cuestionar y repensar las ideologías dominantes y el modo en cómo las personas construyen otras alternativas. Partir de lo personal, de la experiencia cotidiana que da sentido a la vida de las personas es un camino para profundizar en el análisis de la realidad social y cultural que incluye lo particular y plural en el seno de lo colectivo.

La segunda cuestión está vinculada con la singularidad que acabamos de plantear; a saber, en narrativa consideramos que la voz de las personas, su historia y su participación está en el centro de la investigación y del proceso educativo. Esto es ya, en sí mismo, una alternativa, en la medida que nos permite comprender que este desafío es ya un modo de transformación (McLaren, 1984). Esta es una posición no siempre compartida por el mundo académico en el que nos encontramos, ya que goza de buenas dosis de incomprensión, que pone de manifiesto cuáles son los criterios y parámetros hegemónicos con los que tenemos que lidiar. La subjetividad como punto de partida de la investigación, tal como la indagación narrativa pone de manifiesto, no es aceptada desde una perspectiva convencional y positivista de la ciencia. Compartimos las palabras de un ilustre ponente en un acto público en una universidad española, dirigido al alumnado de un máster que recién se iniciaba: 
... tengan cuidado con la línea de investigación a seguir porque la investigación cualitativa, incluso una que se está poniendo de moda, la narrativa, no puede demostrar nada a ciencia cierta frente una investigación de tipo cuantitativo, ya que la investigación cuantitativa sí puede dar unos resultados certeros mientras que desde el otro paradigma simplemente son resultados que tenemos sin más.

Conceptos como resultados, demostración, certeza, verdad, objetividad, etc., no son considerados desde la perspectiva narrativa en la medida que distancian la realidad de los sujetos que la construyen y carecen de potencial transformador. Sitúan el conocimiento más allá de los sujetos que actúan dejando fuera el compromiso y la responsabilidad como componentes propios de la investigación. El giro narrativo de los 80 justamente se sitúa en esta posición, poniendo en valor la construcción colectiva de la realidad, la experiencia como conocimiento, la centralidad del sujeto y la visión narrativa de la ciencia. Cuando hablamos del cuerpo nos situamos en lo personal, en el espacio que nos relaciona con el mundo y con los otros y que nos constituye en tanto que es a través del mismo que se produce la experiencia. Por tanto, es el lugar originario donde se generan las condiciones para la transformación personal y social. Se da la paradoja de que a pesar de esto, desde el punto de vista académico ha permanecido oculto, o como mucho, mediado por parámetros externos, como el rendimiento, la competición, la medida, etc.

La literatura muestra la importancia de la narrativa como un enfoque desde el que abordar ciertas cuestiones sociales y culturales (Becker,1996; Bertaux, 1981; Chase 2015; Denzin, 1970; Dotta y Lopes, 2013; Plummer, 1983). A partir de los estudios feministas y culturales que rompieron la división entre ciencia y política (Harding, 1996) y lo que autores como Norman Denzin (2003) y Jean Clandinin (2007) han denominado "giro narrativo o la vuelta al uso de la narrativa", se ha hecho necesario recuperar cuestiones referidas a la identidad y a la subjetividad como elementos centrales de la investigación narrativa, que nos permitan comprender las dinámicas socioeconómicas y culturales que construyen, en nuestro caso, la realidad educativa (Redon y Ángulo, 2017). Recuperar esta perspectiva subjetiva de la investigación, además de incluir a la persona participante como eje central del proceso, supone comenzar a re(pensar) al investigador/a como un elemento más de todo el proceso relacional de la investigación. Ello implica considerar la subjetividad como una condición necesaria para el conocimiento social y para dar significado a lo construido en la relación investigativa (Bruner, 1997; Ricoeur, 1995).

Las ciencias del deporte y la educación física no quedan al margen de esta condición personal, subjetiva y socio-cultural, máxime si empezamos a considerar, como venimos defendiendo, que todo lo relativo al cuerpo y al movimiento no es un fenómeno externo a lo que sucede en y al cuerpo, sino que da sentido -también simbólicamente- al proceso de humanización (Merleau Ponty, 1975). En este sentido, Smith y Sparkes (2009a, p. 3) refiriéndose a la importancia de la narrativa señalan que este tipo de investigación ayuda a "quitar de los ojos el velo del convencionalismo, ya que las historias dejan asomar sutilmente preguntas, a veces inquietantes, sobre las prácticas relacionadas con el deporte y el ejercicio".

La tradición hasta nuestro días en el ámbito deportivo y de la educación física en contextos escolares y educativos, salvo experiencias puntuales, tiene eminentemente un carácter instrumental, instructor, clasificador y de disciplina deportiva. Como apuntan Prados, Márquez y Padua (2017), en la formación inicial del profesorado y en las ciencias del deporte se ha obviado a lo largo de su historia el significado de lo corporal como elemento constitutivo del "ser docente", a la vez que se ha excluido a las personas -estudiantes, deportistas, educadores, técnicos, 
profesionales, etc.- como referentes centrales de la experiencia. Bien es cierto que en los espacios curriculares, aunque minoritariamente, el cuerpo ha ganado un espacio relevante a través de asignaturas propias del ámbito de Educación Física en la formación del profesorado y algo más en la especificidad del grado en Ciencias de la Actividad Física y el Deporte, en la universidad española.

Es importante considerar que todo lo relativo al entrenamiento, acondicionamiento físico, biomecánica del movimiento, aspectos médicos y fisiológicos, etc. se adhiere a una forma concreta de entender lo físico - corporal que pone en valor solo los efectos que produce, los modos de mejorarlo, el rendimiento más adecuado, etc. Desde nuestro punto de vista, las tradiciones biomédicas, físicas, biomecánicas, neurobiológicas, etc. abordan lo corporal como una cuestión entrenable, medible y controlable; por tanto externa al sujeto mismo, salvo por sus resultados. Desde otra posición ideológica y epistemológica, de corte socio-constructivista, lo corporal hace referencia a cómo el ser humano va construyendo el sentido de quién es a través de sus experiencias corporales, aspectos que se han obviado en la perspectiva anterior.

En la actualidad emergen cuestiones relativas a lo emocional y afectivo, y a los procesos neuroeducativos que acontecen en el aprendizaje que ha sido motivo de avance y profundización en diferentes ramas y disciplinas de estudio. Sin embargo, una vez más, en relación con lo corporal el tratamiento de estas cuestiones se centra en mediciones y resultados de entrenamientos acerca del rendimiento físico. La construcción de nuestro ser corporal en relación ha de ser una preocupación que nos haga comprender que el cuerpo "habla" de quiénes somos y de cómo expresamos mientras aprendemos. Planella (2006) denomina a esto cuerpo encarnado, aquél que habla de la subjetividad del sujeto situado en contexto, de sus modos y maneras de contarse en relación. Somos un cuerpo que expresa, que se afecta, que se emociona, que es y necesita procesos de comunicación que den sentido a la identidad y subjetividad de cada persona y en cada situación... Es por ello, que las narrativas, en tanto relatos de las personas situados en contexto, nos dan claves para ir construyendo un conocimiento compartido de lo que el cuerpo en movimiento aporta a los procesos educativos y formativos.

A partir de esta presentación, es pertinente profundizar sobre lo que para nosotros, y dentro del grupo de investigación ProCIEi, significa la investigación narrativa con trayectorias de vida de las personas a través de sus biografías, relatos e historias de vida. Planteamos este enfoque en la medida que nos permite adentrarnos en la vida y experiencias de las personas desde sus voces y en los modos que tienen de expresar sus vivencias, como el modo de comprender el modo como se han construido como sujetos y de esta forma como están siendo parte del proceso de construcción del mundo (Rivas, Leite y Prados, 2014). O dicho de otro modo, conocemos el mundo, la sociedad, las instituciones a través de comprender a las personas y el modo como nos cuentan sus vidas. En este sentido, entendemos la realidad como una construcción colectiva analizada e interpretada desde la óptica de lo identitario, del contexto social, político y cultural, intentando explicarnos el mundo en el que vivimos e interpretándolo desde una perspectiva dialógica. Desde esta perspectiva se supera la dicotomía entre lo subjetivo y lo institucional (Rivas, 2009; Rivas y Herrera, 2009) compartiendo y construyendo un saber horizontal, práctico, relacional, emocional y sociopolítico con los diferentes "actores" de la investigación (Márquez, Padua y Prados, 2017).

En relación al trabajo que desarrollamos en nuestras aulas, el aspecto más relevante que nos permite afrontar esta dimensión crítica y constructivista del cuerpo, es la dimensión experiencial. Desde la propuesta de Conle (2014), en 
particular, la experiencia nos pone en condiciones de comprender el cuerpo y el movimiento en su totalidad. Esta autora plantea que al trabajar con narrativas, con historias de vida, biografías o relatos, se nos permite dar profundidad a lo que indagamos. Esta hondura aporta matices, detalles, certezas y también incertidumbres... que dan sentido a lo que vivimos; lo cual abre la puerta a poder indagar y desplegar significados quizá insospechados a primera vista. Siguiendo un principio conocido desde la investigación etnográfica, se trata de hacer extraño lo familiar, creando condiciones para la comprensión crítica de nuestra realidad. Bien es cierto que el cuerpo ha estado presente en los estudios de educación física y deportiva pero tradicionalmente tratados casi en su totalidad y hasta la actualidad con una visión cartesiana, atomizada y dualista. Desde esta perspectiva se considera lo motriz y lo corporal como algo imperfecto, como un espacio inacabado que hay que cuidar y entrenar. Sin embargo, una atención o escucha atenta de lo que acontece en el cuerpo, de los entresijos y significados que las personas dan a lo que sucede en sus cuerpos, en sus entrenos, por ejemplo, esta visión o bien es inexistente o bien queda en una plano marginal. La propuesta de narrar el cuerpo desde la experiencia y desde el sentido y significados que las personas le otorgan nos adentra en otro tipo de relación y de conocimiento que puede

hacer posible saber más sobre nosotros mismos o sobre los contextos en los que estamos (...) la dimensión corporal de la docencia ha pasado desapercibida, en general en la investigación y en particular en la formación inicial y permanente (...); difícilmente consideramos la docencia como una profesión corporal (Martínez y González, 2016, p. 260).

Nuestra trayectoria (particularmente la de Esther Prados) nos sitúa en la formación inicial, tanto en los grados de Magisterio, en la especialidad de Educación Física, como en la formación específica de alumnado en Ciencias de la actividad física y el deporte. La experiencia y trayectoria de años de docencia e investigación apuntan a que estos espacios y tiempos en la formación inicial, si por algo se definen, es por la presencia de un cuerpo tremendamente dirigido y una gran ausencia de un cuerpo sensorial. En sus estudios, De Mattos, Prados y Padua (2013) han puesto de manifiesto que el alumnado

hace cosas con sus cuerpos, poseen esquemas cognitivos que fomentan sobre todo la, automatización y la repetición. Desarrollan prácticas motrices estereotipadas, pensando y aspirando al movimiento enérgico, técnico y eficiente. Incorporar al análisis de lo gestual, lo experimental, la capacidad para expresarse con una gama amplia de movimientos y experiencias es un reto pendiente (p.64)

De acuerdo a esto nuestro planteamiento, como dice Conle (2014) no sólo aspira a plantear situaciones problemáticas, de dificultades o de cuestiones que va mal, sino de situaciones cotidianas, de relatos corporales o narrativas deportivas que al situarlas contextualmente y compartirlas permiten comprender(nos), así como buscar y ampliar el horizonte sobre la corporeidad. Las narrativas compartidas permiten presentar múltiples conexiones entre sí, establecer elementos para la reflexión y el análisis, así como indagar en la construcción de identidades. Dado que la corporeidad, es decir, lo relativo a lo corporal, emocional y cultural desde una contextualización de las experiencias y prácticas corporales yace y se construye en nuestra condición humana en tanto que relacional, las narrativas corporales constituyen un espacio donde lo narrado, contado y compartido hablará de los diferentes modos poliédricos en los que cada quién encarna su experiencia personal, formativa, deportiva o profesional. De este modo se da sentido a los 
"cuerpos encarnados" de Planella (2006) que definíamos anteriormente, según el cual cualquier historia contada contiene al mismo tiempo al cuerpo como causa, motivo, medio o expresión. Esto permite considerar que la experiencia vivida en el cuerpo tiene que ver con lo que Contreras (2013, p.125) denomina el saber de la experiencia, es decir, alejarse de la experiencia que considera "el saber como acumulación de conocimiento práctico para adentrarse en un modo, siempre en movimiento, de preguntarse por el sentido de lo vivido y de lo que significa y supone la relación educativa, siempre atravesada por la cuestión de la alteridad".

La propuesta que presentamos está estrechamente relacionada con el uso de la narrativa, a través de relatos biográficos corporales y de dinámicas de conciencia corporal en la formación inicial. Desde las experiencias del alumnado, estas narrativas dan la posibilidad de recuperar y tomar conciencia de los significados subjetivos que cada persona confiere a sus aprendizajes a lo largo de su vida escolar y académica y de cómo ello constituye modos de pensar, hacer, vivir y construir conocimiento acerca de la motricidad humana y sus diferentes significados culturales y educativos. Lo interesante de estas narrativas es (dar)se cuenta del cuerpo como experiencia y no del cuerpo como cosificación, objeto o medio. El cuerpo como experiencia incluye per sé lo afectivo, lo emocional, lo relacional... como elementos constitutivos del ser docente, del ser deportista, del ser entrenador/a, del ser estudiante, del Ser humano. Expresado de otro modo, Smith y Sparkes (2009a, p. 5), refiriéndose a ello, nos dicen que "nuestra vida gira en torno a nuestro cuerpo. Dependemos de él, nos relacionamos con el mundo y las personas a través de él. Lo sentimos y sentimos con él. Existimos con él y, en ocasiones, para él".

\section{Los relatos corporales en la formación inicial}

Para un campo de conocimiento como es la educación física y la motricidad humana en sus más amplias manifestaciones relacionadas con el movimiento y la actividad física y deportiva, se hace extraño abordar cuestiones formativas, reflexivas e investigadoras acerca de las mismas, a partir de una mirada narrativa y subjetiva de la experiencia. La extrañeza no viene condicionada tanto por el hecho de analizar el movimiento en sí, como el de abordar aspectos que vinculen el movimiento a significados emocionales y culturales que las personas otorgan a lo que viven. Como apuntábamos al inicio de este escrito son incipientes los estudios o experiencias dentro del ámbito de las ciencias de la actividad física y el deporte que profundicen en el sentido ontológico que las propias personas otorgan a su práctica. Sin poner en cuestión algunas máximas populares como, "el deporte es bueno para la salud", "la actividad física es vitalmente necesaria para el desarrollo humano y social", "mens sana in corpore sano", "tu cuerpo es el reflejo de lo que haces con él", "sin el deporte no podría vivir"...hoy sabemos también -sobre todo a partir del desarrollo de la neurociencia- que nuestro cuerpo es representado y construido por el mapa de nuestra biografía personal, en la que confluyen multiplicidad de aspectos y de condiciones socioculturales. Lo educativo ha conformado desde nuestros inicios un modo de acceder, sentir y construir nuestras "huellas corporales"; es decir, esa forma de ser y tener un cuerpo en tiempo presente (Martínez y González, 2016), que se nos ha ido configurando no sólo por experiencias deportivas o de movimiento, sino también por toda una amalgama de experiencias que hemos ido internalizando y que conforman nuestra epigenética celular y relacional.

Las trayectorias a lo largo de la vida escolar constituyen un foco importante de estudio en nuestra experiencia narrativa. Las biografías y relatos escolares que tienen como foco lo corporal constituyen una herramienta de aprendizaje, reflexión 
y crítica importante en la formación inicial del alumnado; a través de los relatos accedemos al imaginario que los y las estudiantes construyen acerca de la idea de ser educadores o educadoras del movimiento, ya que en sus historias se pone de manifiesto esquemas prácticos para la toma de decisiones, tanto en su formación como en su futura profesión. Cortés at. col. (2015) sostienen que estos esquemas son el resultado de la conjunción de múltiples elementos que configuran un conjunto de representaciones que rigen las prácticas y modos de aprendizaje en torno a corporalidad y el movimiento. Las representaciones que construye el alumnado se vinculan con sus trayectorias deportivas, con sus biografías escolares, su formación inicial, su socialización laboral y con la idea de cuerpo, movimiento y deporte que han vivido. Como sostienen estos autores, las informaciones 0 representaciones que el alumnado tiene no son necesariamente accesibles a la conciencia. Por ello, el uso de biografías o relatos corporales en la formación inicial -sabiendo que las biografías y relatos representan la evocación de experiencias pasadas en el momento presente- permiten reflexionar acerca del bagaje experiencial, cultural y educativo en diferentes contextos y etapas de aprendizaje, a través de los cuales explicitan paulatinamente el contenido de las representaciones y las actitudes de la persona en relación a la idea de lo que significa un cuerpo educado deportiva y motrizmente.

Utilizar estos instrumentos como medio de formación en su paso por la etapa universitaria ayuda a que el alumnado comprenda y acceda a los diferentes elementos ideológicos que se ponen en juego en la realidad y en la comprensión actual de los diferentes modelos corporales y deportivos. Además se pone de manifiesto que a través de los relatos el alumnado puede adquirir elementos de reflexión, análisis e interpretación que ellos y ellas hacen de su proceso formativo y comprender que ello no es simplemente un producto de un discurso intelectual, "científico" y académico sobre lo deportivo y lo corporal, sino fruto de las propias tradiciones culturales y de los marcos de comprensión que los y las estudiantes tienen. La toma de conciencia por parte del alumnado, tanto del imaginario como de la experiencia en torno a lo corporal, representa una puerta abierta entre lo implícito y lo explícito que contiene el conocimiento sobre esta materia, al tiempo que permite identificar elementos considerados constitutivos en las funciones, roles y prácticas que implica la enseñanza de la educación física y el deporte.

Del mismo modo, utilizar el relato corporal como constitutivo del proceso de interacción relacional en el aula, ayuda a representar un proceso comunicativo centrado en la persona y en las formas en las que edifican colectivamente su visión del mundo y de su realidad; es decir, su perspectiva peculiar acerca de lo que significa la educación física, el deporte y sus múltiples manifestaciones. Esta práctica docente formativa desde el punto de vista narrativo no representa constructos teóricos preestablecidos y cerrados, sino más bien nos muestra un proceso en marcha, versátil, en movimiento. Por tanto, siguiendo a Rivas (2009) estamos ante un modo de participación colectiva en la historia del conocimiento, a partir de nuestra entrada (del alumnado) en la misma en un momento dado. De este modo, lo que el alumnado relata acerca de su experiencia corporal y deportiva no es sólo un conjunto de hechos o acontecimientos, sino fundamentalmente, su comprensión de sus vivencias, de los procesos de aprendizaje y el modo cómo lo han reelaborado y convertido en una condición para su acción futura. En los relatos biográficos están presentes las condiciones en que éstos se han producido, los contextos en que han actuado y la elaboración personal que ha hecho de todo este conjunto de datos. Esto supone dar valor a los sujetos participantes como 
portadores de sentido y de contenido (Prados, Márquez y Padua, 2017), a la vez que permite generar procesos de reflexión acerca de qué, cómo, por qué y para qué se enseña y aprende en su formación inicial.

\section{Narrativas de lo corporal}

Las aportaciones de Damasio (2009) ponen de manifiesto cómo las experiencias, recuerdos y reacciones dejan huellas, trazas y expresiones corporales que el cuerpo graba y que conforman la plasticidad, movimiento y transformación de nuestro bagaje neuronal, y por tanto, relacional. Nuestro desarrollo es fruto de un proceso interactivo, es decir, una actividad relacional entre un agente (el cuerpo) y el medio contextual dotando a dicha interacción de "creación de sentido" (De Bruin y Kästner, 2012). La búsqueda de este sentido de lo que acontece en el cuerpo, Cantero (2017) la denomina "traer el cuerpo presente" a la experiencia in situ del aula; es decir, hacer del conocimiento una experiencia corporeizada, integrar conocimiento y acción desde una contextualización de los diferentes aspectos biopsicosociales que se ponen en juego en la experiencia humana. De ahí que narrar lo que acontece en la acción, integrando aspectos físicos, cognitivos y emocionales, junto al imaginario social, puedan darnos razón de la complejidad e incertidumbre que contiene la experiencia humana.

Cuando le solicitamos al alumnado que relate alguna de las experiencias que se han quedado "grabadas" en su cuerpo, o que recuperen algún hecho que marcó o determinó un hito en su vida deportiva, o que rescate memorias, detalles o anécdotas de como vivió algunas de las experiencias escolares donde el movimiento o el cuerpo fuese central, irrumpen en sus memorias aspectos que nos hacen pensar sobre el sentido y significado que el cuerpo ha tenido a lo largo de su vida. Ello nos hace recuperar al alumnado como sujeto pedagógico, más allá de considerar al cuerpo objetual como cuerpo educable y así adentrarnos en la conciencia íntima del cuerpo como ser vivido, como un cuerpo educando (Gervilla, 2000).

La experiencia en el aula universitaria con el uso de narrativas corporales a través de relatos escritos y de performance expresivo corporales, representa una novedad en el aprendizaje de la disciplina de la educación física y deportiva. Sin embargo, el alumnado manifiesta ciertas resistencias a escribir desde la subjetividad acerca de sus experiencias. En buena parte esto es así, porque lo subjetivo está asociado a la desvaloración personal, a la falta de confianza en uno mismo, al hecho de considerar que lo personal no tiene tintes de objetivad y por tanto no es considerado como un conocimiento académico. También por el miedo a ser calificados o enjuiciados en aspectos tan personales e íntimos.

De igual modo, narrarse expresivamente a través de performance corporales pone de manifiesto la dificultad que entraña situarse en público a través del lenguaje no verbal del cuerpo -a pesar de que lo deportivo también supone dicha exposición-, así como la falta de habilidades en la comunicación no verbal, el temor a exponerse y comunicarse con los demás, el sentimiento de vergüenza y ridículo a que los demás te vean y califiquen o la dificultad de expresar emociones y sentimientos a través del cuerpo. Estas disyuntivas nos acercan a algunos de los significados que rodean las concepciones construidas a lo largo de décadas sobre lo corporal y lo motriz y a de-construir o cuestionar la forma tradicional de entender lo corporal como un universo exclusivo de disciplinas tradicionalmente consideradas específicas de este conocimiento como son las del campo biomédico, biomecánico o deportivo. Rescatamos para explicar este matiz el testimonio de una alumna, que resulta paradigmático de este temor acuñado biográficamente en nuestro cuerpo 
tengo dificultad en expresarme sólo con movimiento libre, así expresivo, como si fuese danza, o con movimientos bellos o quizá más estéticos, no sé, si haces deporte en una cancha o compites delante de mil personas no lo viven como una exposición al otro sino como una exhibición o competición. En estos contextos no te "desnudas" al otro, no te miran a ti, te miran como un deportista y no como una persona que tiene sentimientos"

Estas narrativas corporales se convierten así en un soporte que contiene los saberes y aprendizajes vividos, al tiempo que son una oportunidad para reflexionar sobre la existencia o no de procesos creativos en torno al cuerpo. Del mismo modo, ayudan al alumnado a descubrir y comprender que a través de todo lo relativo a la expresividad corporal y humana se puede reflexionar sobre cuestiones educativas, y así, vincular la propia experiencia corporal con aprendizajes adquiridos y con los modos y estructuras establecidas en la enseñanza -cuerpo obediente, mecanizado, reglado, normativizado, comparado, calificado, etc.- (Prados, Márquez y Padua, 2017). Por otra parte, las narrativas personales, particulares, ponen de relieve experiencias únicas, con rostros y cuerpos que hablan de situaciones de dolor, sufrimientos, superación, retos, dificultades, marginación o exclusión, y de esta forma contrastar los discursos que las instituciones hacen sobre las grandes historias o grandes épicas que el ser humano es capaz de hacer con su cuerpo, con el deporte.

El análisis sistematizado de estas narraciones nos lleva a exponer algunas de las dimensiones interpretativas que nos ayudan a comprender cuales son las "fuentes" de las que se han aprehendido los discursos, los modelos y las prácticas del cuerpo en movimiento y que traspasan los muros de la academia en la formación inicial del alumnado. Uno de los significados más presentes en sus narraciones se refiere a la ausencia de la dimensión expresiva y emocional en su aprendizaje. La formación recibida es eminentemente racional y menosprecia la experiencia personal fruto de metodologías tradicionales, instructivas, memorísticas y pasivas que inciden sólo en la trasmisión de contenidos y prácticas corporales estandarizadas. Derivada de esta cuestión, encontramos la eterna separación cartesiana: una cosa es el cuerpo físico y otra bien distinta el cuerpo emocional y cognitivo, de ahí que las concepciones para entrenar al cuerpo se alejan, invisibilizan o reprimen al cuerpo que piensa o se emociona mientras se entrena. El entrenamiento corporal lejos de ser un espacio para el aprendizaje es un espacio para competir con uno mismo, buscar éxitos o records en los que sólo el sacrificio y el esfuerzo representan la voluntad del ejercitante.

Por otra parte, las narraciones plantean cuestiones que sugieren la necesidad de hablar del cuerpo como un constructo que se ha forjado a base de condicionamientos sociales de los cuáles es difícil desprenderse. Los relatos y las performances del alumnado dejan entrever elementos que nos permiten hacer del cuerpo un espacio de convergencia discursiva en tono a lo que hemos denominado "cuerpos dietéticos, cuerpos sufridos, cuerpos imaginados y cuerpos lenguajeados" (Prados, 2004). En términos dietéticos el cuerpo es un espacio para la búsqueda de la belleza a través de la higiene, alimentación, moda y cultura física. Están presentes en esta búsqueda "la ansiada eterna juventud", el poder, el éxito y la seducción. La preocupación por la salud, sin embargo, deja entrever que ciertas prácticas con el cuerpo buscan la reproducción de determinados cánones de belleza, ocultando dificultades, riesgos o insatisfacciones con el propio cuerpo. La búsqueda de esta belleza se nutre de imágenes y proyecciones de la cultura del bienestar y consumo que atraviesa al propio cuerpo, idealizándolo y deseando ese 
cuerpo imaginado. La idealización de un cuerpo saludable, fuerte, sano, perfecto, atractivo.

Este cuerpo imaginado al que hay que aspirar de acuerdo al planteamiento hegemónico, expresa muy poco o nada acerca del mundo interior de la persona, de su sensibilidad, sus formas de pensar y emocionarse, sus sentimientos y sus pasiones. La experiencia narrada del cuerpo "deportivizado" lleva inherente el sufrimiento. No hay records sin dolor, sin llegar a los límites. El cuerpo sufrido nos adentra en la creencia que el cuerpo es un lugar de lucha, de combate, de rendimiento, de eficacia y eficiencia, alejando, una vez más, la posibilidad de vivirlo como lugar de integración, donde cabe incluir emoción, goce y disfrute.

Finalmente, recuperar experiencias pasadas buscado matices, sensaciones, recuerdos y memorias nos permite acercarnos a un cuerpo lenguajeado, aquel que ayuda a percibir que el cuerpo es plataforma de expresiones sociales, culturales y personales. El cuerpo no es sólo lo que se ve, sino también lo que guarda, esconde o dice con su expresión, con sus propias formas de lenguaje no verbal. De ahí que las historias personales nos hablen de un caleidoscopio de superación, trasgresión y reconocimiento en la diversidad. Hay muchos modos de vivir la corporalidad, y por tanto, diversidad en las maneras de construir la propia identidad corporal. Las historias del alumnado nos invitan a la búsqueda de matices en sus rostros, en sus gestos, en sus propios movimientos y prácticas deportivas, aquellas que los alejen de estereotipos clásicos, para adentrarse en los que cada uno quiere ser o le dejen ser.

\section{Narrativa de la escucha corporal -a modo de conclusión-}

Lo que tratamos de proporcionar en la formación universitaria a través de las narrativas corporales del alumnado está vinculado a dos cuestiones fundamentales: una, escuchar, escribir, compartir y generar creativamente conocimiento dentro del grupo desde un principio de horizontalidad para establecer dinámicas en el aula universitaria, aún por descubrir, que favorezca la escucha interior de la otra/o. En este sentido hablar sobre el cuerpo y desde el cuerpo, contar de sí y sobre sí hace presente la escucha interior y la escucha del otro. $Y$ dos, narrarse junto a otros y otras, nos remite no sólo al hecho de pensar el cuerpo desde las habilidades o capacidades físicas, desde las potencialidades del entrenamiento o desde la capacidad de ejecución técnica o táctica, entre otras, sino también a la importancia y necesidad que el ser humando tiene de comprender qué le sucede, que siente en su cuerpo mientras hace, ejecuta, se mueve, pierde, gana, triunfa o fracasa. Es ahí, en estas experiencias, donde se imbrican "las pequeñas historias que dan cuerpo al cuerpo".

\section{Bibliografía}

Barbero, J.I. (2006). Ficción autobiográfica en torno a la cultura corporal y la vida cotidiana. Educación Física y Deporte, Medellin, V. 25, núm. 2, 47-63.

Berry, K. S. (2008). Lugares (o no) de la pedagogía crítica, en P. McLaren, J.L. Kincheloe (eds.) Pedagogía crítica. De qué hablamos, dónde estamos (pp. 117140) Barcelona: Grao

Becker, H. S. (1996). The epistemology of qualitative research, en Jessor, R., Colby, A. y Schweder, R. (Eds.), Ethnography and human development: Context and human developement (pp. 53-71). Chicago: University of Chicago Press.

Bertaux, D. (1981). El enfoque biográfico: su validez metodológica, sus potencialidades. Cuadernos de Ciencias Sociales: Historia oral e historia de vida, núm. 18, 55-80. 
Bruner, J. (1997). La educación puerta de la cultura. Madrid: Visor Dis. S. A.

Chase, S. (2015). Investigación narrativa. Multiplicidad de enfoques, perspectivas y voces, en Denzin I. y Licoln N. (coord.) IV Manual de Investigación narrativa. Métodos de recolección y análisis de datos. Barcelona. Gedisa.

Cantero, M.A. (2017). ¿Un "cuerpo presente" en la escuela? Una propuesta: time in, Prados, M.E., Márquez. M.J. y Padua, D., Otra pedagogía en movimiento. Experiencias educativas en la formación inicial (En prensa). Editorial Universidad de Almería.

Clandinin, J. (2007). Handbook of Narrative Inquiry: Mapping a Methodology

Clandinin, J. (2013). Engaging in Narrative inquiry, CA: Left Coast Press

Conle, C. (2014). Anatomía del currículum narrativo, en Rivas. J.I., Leite, A. y Prados, M.E. (coord.), Profesorado, escuela y diversidad. La realidad educativa desde una mirada narrativa. Málaga: Ediciones Aljibe.

Contreras, J. (2013). El saber de la experiencia en la formación inicial del profesorado. Revista Interuniversitaria de Formación del Profesorado, 78 (27,3), 125-136.

Cortés, P. at. Col. (2015). La voz del alumnado en formación. Revista digital Cuadernos de Pedagogía, núm. Marzo. Editorial Wolters Kluwer, ISBN-ISSN: 23866322.

Damasio, A.R. (2009). El error de Descartes: la emoción, la razón y el cerebro humano. Barcelona: Crítica

De Bruin, L. y Michael, J. (2016). Prediction error minimization: Implications for Embodied Cognition and the Extended Mind Hypothesis. Brain and Cognition (en prensa).

De Mattos, B., Prados, E. y Padua, D. (2013). A voz dos alunos (as): Uma investigaçao narrativa sobre o que sente, pensa, diz e faz o alunado de educaçao físca em sua formaçao inicial. Movimento. Revista de Educaçao Física da FURGS, 19 (4), 251-269.

Devís, J. y Sparkes, A. (2001). La crisis de identidad de un estudiante universitario de educación física. Un estudio biográfico, en Devís, J. (Coord.). La educación física, el deporte y la salud en el siglo XXI. Alcoy: Marfil.

Devís, J. y Sparkes, A. (2004). La crisis de identidad de un estudiante universitario de educación física. La reconstrucción de un estudio biográfico, en Sicilia, A. y Fernández-Balboa, J.M. (coord.). La otra cara de la investigación. Reflexiones desde la educación física. Sevilla: Wanceulen.

Denzin, N. (1970). The research act. Chicago: Aldine Publishing

Denzin, N. (2003). Foreword: narrative's momento, en Andrews, M., Sclater, S., Squire; C. y Treacher, A. (Eds.), Lines of narrative. Londres: Routledge, 2003.

Dotta, L.T. y Lopes, A. (2013). Investigaçao narrativa, formacçao inicial de professores e autonomia dos estudantes: urna revisao de literatura Narrative. En Revista Educación y Futuro, (29), 129-155.

Eisenberg, R. (coord.) (2007). Corporeidad, Movimiento y Educación Física, 1992-2004. Estudios conceptuales. México: Consejo Mexicano de Investigación Educativa, № 12 (1).

Harding, S. (1996). Ciencia y feminismo. Madrid: Ediciones Morata, S. A.

Fernández-Balboa, J.M. y Prados, M.E. (2014). The conscious system for the movement technique: an ontological and holistic alternative for (Spanish) physical education in troubled times. Sport, Education and Society, 19 (8), 10891106.

Gervilla, E. (2000). Valores del cuerpo educando. Antropología del cuerpo y educación. Barcelona: Herder

Hernández, F. (2011). Las historias de vida en el marco del giro narrativo en la investigación en Ciencias Sociales: los desafíos de poner biografías en 
contexto . En Hernández, F; Sancho, J.M. y Rivas, J.I. (eds). Historias de vida en educación. Biografías en contexto. Barcelona: Esbrina - Recerca, Universitat de Barcelona http://hdl.handle.net/2445/15323

López, V., García, A., Pérez, D., López, E. y Monjas R. (2004). Las historias de vida en la formación inicial del profesorado de educación física. Revista Internacional de Medicina y Ciencias de la Actividad Física y el Deporte, Madrid, núm. 4, n.13, p. 45-57.

Márquez, M. J.; Prados, M. E. y Padua, D. (2013). "El uso de la biografía en el aula universitaria. Tres experiencias en diálogo", en Lopes A., Hernandez, F, Sancho, J. y Rivas J.I. (coords.) Historias de vida em educaçao. A contruçao do conhecimento a partir de histórias de vida. Barcelona: Creative Commons. Deposit digital: http://hdl.handle.net/2445/47252.

Márquez, M. J.; Prados, M. E. y Padua, D. (2014). Relatos escolares y construcción del currículum en la formación inicial del profesorado. Bioeducamos. Revista Tendencias Pedagógicas. Monográfico Las Historia de Vida, nçum. 24, 113132.

Márquez, M.J., Padua, D. y Prados, M.E. (2017). Investigación narrativa en Educación. Aspectos metodológicos en la práctica, en Redon, S. y Angulo, F. Investigación cualitativa en Educación. Buenos Aires: Miño Dávila.

Martínez, L. y González, G. (2016). Docentes de carne y hueso. Enseñar con cuerpo. Revista Ágora para la EF y el Deporte, núm. 18 (3), 259-275.

McLaren, P. (1984). La vida en las escuelas. Una introducción a la pedagogía crítica en los fundamentos de la educación. México: Siglo XXI.

Merlau-Ponty, M. (1975). Fenomenología de la percepción. Barcelona: Península.

Mourao, I. y Morel, M. (2006). As narrativas sobre o futebol feminino: o discurso da midia impressa em campo. Revista Brasileira de Ciencias do Esporte, São Paulo, v. 26, n. 2, p. 73-86.

Padua, D. y Prados, M.E. (2014). El uso de autobiografías en la formación inicial de los futuros docentes. Dos experiencias en la Universidad de Almería, en Rivas, J.I., Leite, A. y Prados, E. Profesorado, Escuela y Diversidad. La realidad educativa desde una mirada narrativa. Málaga: Algibe.

Pavía, V. (2001). Una invitación a escribir historias de la propia práctica. Lecturas: educación física y deportes, Buenos Aires, v. 7, n.40, p 1-2. Brouwer.

Planella, J. (2006). Cuerpo, cultura y educación. Bilbao: Desclée de

Plummer, K. (2005). Documents of life. And invitation to a critical humanism. London: Sage Pub.

Prados Megías, M. E. (2004). El cuerpo en clave de mujer. Revista Meridiam. Instituto Andaluz de la Mujer, 33, 54-57.

Prados, M.E., Márquez, M.J., Padua, D. (2017). El cuerpo como experiencia. Una propuesta narrativa, expresiva y corporal en la formación inicial del profesorado, en Pérez. M. y Rodríguez, J. (coords.) Buenas prácticas docentes del profesorado universitario. Barcelona: Octaedro.

Prados, M.E., Márquez, M.J., Padua, D. (2017). Life stories as a biographicnarrative method. How to listen to silenced voices. Revista Procedia and Behavioral Sciences, 237, 962-967.

Redon, S. y Angulo, F. (2017). Investigación cualitativa en Educación. Buenos Aires: Miño Dávila.

Ricoeur, P. (1995). Tiempo y narración 1. Configuración del tiempo en el relato histórico. México: Siglo XXI. 
Rivas, I. (2009). Narración, conocimiento y realidad. Un cambio de argumento en la investigación educativa, en J. I. Rivas y Herrera, D. (eds.) Voz y educación. La narrativa como enfoque de interpretación de la realidad. Barcelona: Octaedro.

Rivas, J.I. (2012). Sujeto, diálogo, experiencia: el compromiso del encuentro. En: Rivas, J. I.; Hernández, F.; Sancho, J. M.; Núñez, C. (coords). Historias de vida en educación. Sujeto, Diálogo, Experiencia. Barcelona: Esbrina - Recerca, Universitat de Barcelona, http://hdl.handle.net/2445/32345

Rivas J.I. y Herrera, D. (2009). Voz y Educación. La Narrativa como enfoque de interpretación de la realidad. Editorial Octaedro. Barcelona.

Rivas, J.I., Leite, A. y Prados, E. (coords.) (2014). Profesorado, Escuela y Diversidad. La realidad educativa desde una mirada narrativa. Málaga: Algibe.

Smith, B. (1999). The Abyss: exploring depression through a narrative of the self. Qualitative Inquiry, Londres, v.5, 264-279.

Smith, B. y Sparkes, A. (2002). Men, sport, spinal cord injury, and the construction of coherence: narrative practice in action. Qualitative Research, Londres, v.2, p.43- 171.

Smith, B. y Sparkes, A. (2005). Men, sport, spinal cord injury, and narratives of hope. Social Science and Medicine, Oxford, v. 61, p.1095-1105.

Smith, B. y Sparkes, A. (2006). Narrative inquiry in psychology: exploring the tensions within. Qualitative Research in Psychology, Londres v.3, p.169-192.

Smith, B. y Sparkes, A. (2007). Sport, spinal cord injury, and body narratives: a qualitative project. Health Psychology Update, Leicester v.16, p.26-33.

Smith, B. y Sparkes, A. (2008a). Contrasting perspectives on narrating selves and identities: an invitation to dialogue. Qualitative Research, Londres v.8, p. 5-35.

Smith, B. y Sparkes, A. (2008b). Changing bodies, changing narratives and the consequences of tellability: a case study of becoming disabled through sport. Sociology of Health and Illness, Boston, v. 30, p. 217-236.

Smith, B. y Sparkes, A. (2009a). Narrative inquiry in sport and exercise psychology: What can it mean, and why might we do it? Psychology of Sport and Exercise, Londres v.10, p. 1-11.

Smith, B. y Sparkes, A. (2009b). Narrative analysis and sport and exercise psychology: Understanding lives in diverse ways. Psychology of Sport and Exercise, Londres, v.10, p.279-288.

Sparkes, A.C. (2002). Telling stories in sport and physical activity. A qualitative journey. Champaign: Human Kinetics.

Sparkes, A.C. (2003). Investigación narrativa en la educación física y el deporte. Ágora para la E.F. y el Deporte, Palencia v. 2-3, p. 51-60.

Sparkes, A.C. (2005). Refections on an embodied sport and exercise psychology, in Stelter, R.; Roessler, K. (Eds.), New approaches to exercise and sport psychology. Oxford: Meyer y Meyer Sport.

Sparkes, A.C. y Devís, J. (2008). Investigación narrativa y sus formas de análisis: una visión desde la educación física y el deporte., en: Moreno, W. (Ed.). Educación cuerpo y ciudad. El cuerpo en las interacciones e instituciones sociales. Medellin: Funámbulos.

Sparkes, A.C.; Douglas, K. (2007). Making the case for poetic representations: an example in action. The Sport Psychologist, Champaign, n.21, p.170-189.

Sparkes, A.C.; Partington, S. (2003). Narrative practice and its potential contribution to sport psychology: the example of flow. The Sport Psychologist, Champaign, v. 17, p. 292-317.

Sparkes, A.C. y Silvennoinen, M. (Eds.) (1999). Talking bodies: Men's 
narratives of the body and sport. SoPhi: University of Jyvaskyla, Finlandia.

Sparkes, A.C. y Smith, B. (2003). Men, Sport, Spinal Cord Injury and Narrative Time. Qualitative Research, Londres, v.3, n.3, p. 295-320.

Sparkes, A.C. y Smith, B. (2009). Judging the quality of qualitative inquiry: Criteriology and relativism in action. Psychology of Sport and Exercise, Londres v. 10, n.5, p. 491-497.

Van Manen, M. (1998). El tacto en la enseñanza: el significado de la sensibilidad pedagógica. Barcelona: Paidós Ibérica.

Vilela, E. (2000). Cuerpos escritos de dolor. Revista complutense de educación, Vol. 11 (2), 83-106.

\section{Notas:}

i ProCIE responde a las siglas del grupo de investigación interuniversitario HUM-619 denominado, Profesorado, Comunicación e Investigación Educativa. Grupo perteneciente a los grupos PAIDI de la Junta de Andalucía- España. 